\title{
On the Representation of Integer Numbers by the sum of Cube roots
}

\section{Prof. Dr. Raja Rama Gandhi and Edigles Guedes}

${ }^{1}$ Resource person in Math for Oxford University Press, Professor in Math, BITS-Vizag.

${ }^{2}$ World order Number Theorist, Pernambuco, Brazil.

e-mail:edigles.guedes@gmail.com

ABSTRACT. We developed some formulas to represent integer numbers as the sum of cube roots.

Lemma 1. If $c=\frac{a}{b}$ and $b \neq 0$, then

$$
a b^{2}+a b^{2} c=b^{3} c+a^{2} b .
$$

Proof. Suppose that

$$
y=1+z y,
$$

then, we can do

$$
z=\frac{t-1}{t}
$$

and

$$
y=t
$$

Let $t=\frac{a}{b}$ in numerator and $t=c$ in denominator, such that $c=\frac{a}{b}$. Therefore,

$$
\begin{gathered}
\frac{a}{b}=1+\frac{\frac{a}{b}-1}{c}\left(\frac{a}{b}\right), \\
\frac{a}{b}=1+\frac{(a-b) a}{b^{2} c}, \\
\frac{a}{b}=\frac{b^{2} c+(a-b) a}{b^{2} c}, \\
a b^{2} c=b^{3} c+a^{2} b-a b^{2}, \\
a b^{2}+a b^{2} c=b^{3} c+a^{2} b .
\end{gathered}
$$

Corollary 1. If $b=\frac{a}{c}$ or $b=a$ or $b=0$ and $c \neq 0$, then $b$ satisfies the equation

$$
c x^{3}-a(c+1) x^{2}+a^{2} x=0 .
$$

Proof. We substitute $x=b$ in Lemmal and use a bit of algebraic manipulation.

Lemma 2. If $c=\frac{a}{b}$ and $b \neq 0$, then

$$
a^{2} b+a b^{2} c^{2}=a^{3}+b^{3} c^{2} .
$$


Proof. Suppose that

$$
y=1+z y^{2},
$$

then, we can do

$$
z=\frac{t-1}{t^{2}}
$$

and

$$
y=t
$$

Let $t=\frac{a}{b}$ and $t^{2}=c^{2}$, such that $c=\frac{a}{b}$. Therefore,

$$
\begin{gathered}
\frac{a}{b}=1+\frac{\frac{a}{b}-1}{c^{2}}\left(\frac{a}{b}\right)^{2}, \\
\frac{a}{b}=1+\frac{(a-b) a^{2}}{b^{3} c^{2}}, \\
\frac{a}{b}=\frac{b^{3} c^{2}+(a-b) a^{2}}{b^{3} c^{2}}, \\
a=\frac{b^{3} c^{2}+(a-b) a^{2}}{b^{2} c^{2}}, \\
a b^{2} c^{2}=b^{3} c^{2}+a^{3}-a^{2} b, \\
a^{2} b+a b^{2} c^{2}=a^{3}+b^{3} c^{2} .
\end{gathered}
$$

Corollary 2. If $b=\frac{a}{c}$ or $b=-\frac{a}{c}$ or $b=a$ and $c \neq 0$, then $b$ satisfies the equation

$$
c^{2} x^{3}-a c^{2} x^{2}-a^{2} x+a^{3}=0 .
$$

Proof. Substitute $b=x$ in Lemma 2 and use a bit of algebraic manipulation.

Theorem 1. For $c \in \mathbb{Z}_{>1}$, then any integer a has the following representation of the two cube roots

$$
\begin{gathered}
a=\sqrt[3]{\frac{1}{2}\left(-\frac{a^{3}(c+1)}{c^{2}}+\sqrt{\left.\frac{a^{6}(c+1)^{2}}{c^{4}}-\frac{4 a^{6}\left(c^{2}+c+1\right)^{3}}{27 c^{6}}\right)}\right.} \\
+\sqrt[3]{\frac{1}{2}\left(-\frac{a^{3}(c+1)}{c^{2}}-\sqrt{\left.\frac{a^{6}(c+1)^{2}}{c^{4}}-\frac{4 a^{6}\left(c^{2}+c+1\right)^{3}}{27 c^{6}}\right)}\right.} .
\end{gathered}
$$

Proof. By Cardano's formula, a root of $x^{3}+p x+q=0$ is given by

$$
x_{1}=\sqrt[3]{\frac{1}{2}\left(-q+\sqrt{q^{2}+\frac{4 p^{3}}{27}}\right)}+\sqrt[3]{\frac{1}{2}\left(-q-\sqrt{q^{2}+\frac{4 p^{3}}{27}}\right)} .
$$


Suppose that $x=a$, and

$$
x^{3}=-p x-q .
$$

By Corollary 1, we obtain

$$
\begin{gathered}
c(-p x-q)-a(c+1) x^{2}+a^{2} x=0 \\
-a(c+1) x^{2}+\left(a^{2}-c p\right) x-c q=0 \\
a(c+1) x^{2}-\left(a^{2}-c p\right) x+c q=0 .
\end{gathered}
$$

By Bhaskara's formula, we find

$$
x=\frac{\left(a^{2}-c p\right) \pm \sqrt{\left(a^{2}-c p\right)^{2}-4 a c q(c+1)}}{2 a(c+1)} .
$$

On the other hand, by Corollary 2 and (2), we obtain

$$
\begin{aligned}
& c^{2}(-p x-q)-a c^{2} x^{2}-a^{2} x+a^{3}=0, \\
& -a c^{2} x^{2}-\left(a^{2}+c^{2} p\right) x+a^{3}-c^{2} q=0 .
\end{aligned}
$$

By Bhaskara's formula, we put

$$
x=-\frac{a^{2}+c^{2} p \pm \sqrt{\left(a^{2}+c^{2} p\right)^{2}+4 a c^{2}\left(a^{3}-c^{2} q\right)}}{2 a c^{2}} .
$$

We compare (3) with (4), and find

$$
-\frac{a^{2}+c^{2} p}{2 a c^{2}}=\frac{a^{2}-c p}{2 a(c+1)}
$$

and

$$
\frac{\left(a^{2}+c^{2} p\right)^{2}+4 a c^{2}\left(a^{3}-c^{2} q\right)}{4 a^{2} c^{4}}=\frac{\left(a^{2}-c p\right)^{2}-4 a c q(c+1)}{4 a^{2}(c+1)^{2}} .
$$

Solving the system of equations above, we get

$$
p=-\frac{a^{2}\left(c^{2}+c+1\right)}{c^{2}}
$$

and

$$
q=\frac{a^{3}(c+1)}{c^{2}}
$$

Therefrom, we substitute (5) and (6) in (1) and let $x_{1}=a$, so we complete the proof.

\section{Examples}

For $a=3$ and $c=2$, then

$3=\sqrt[3]{\frac{1}{2}\left(-\frac{81}{4}+\frac{15 i \sqrt{3}}{2}\right)}+\sqrt[3]{\frac{1}{2}\left(-\frac{81}{4}-\frac{15 i \sqrt{3}}{2}\right)} ;$ 
for $a=3$ and $c=3$, then

$3=\sqrt[3]{\frac{1}{2}\left(-12+\frac{70 i}{3 \sqrt{3}}\right)}+\sqrt[3]{\frac{1}{2}\left(-12-\frac{70 i}{3 \sqrt{3}}\right)} ;$

for $a=4$ and $c=2$, then

$4=\sqrt[3]{\frac{1}{2}\left(-48+\frac{160 i}{3 \sqrt{3}}\right)}+\sqrt[3]{\frac{1}{2}\left(-48-\frac{160 i}{3 \sqrt{3}}\right)} ;$

for $a=4$ and $c=3$, then

$4=\sqrt[3]{\frac{1}{2}\left(-\frac{256}{9}+\frac{4480 i}{81 \sqrt{3}}\right)}+\sqrt[3]{\frac{1}{2}\left(-\frac{256}{9}-\frac{4480 i}{81 \sqrt{3}}\right)}$

for $a=5$ and $c=2$, then

$5=\sqrt[3]{\frac{1}{2}\left(-\frac{375}{4}+\frac{625 i}{6 \sqrt{3}}\right)}+\sqrt[3]{\frac{1}{2}\left(-\frac{375}{4}-\frac{625 i}{6 \sqrt{3}}\right)} ;$

for $a=5$ and $c=3$, then

$5=\sqrt[3]{\frac{1}{2}\left(-\frac{500}{9}+\frac{8750 i}{81 \sqrt{3}}\right)}+\sqrt[3]{\frac{1}{2}\left(-\frac{500}{9}-\frac{8750 i}{81 \sqrt{3}}\right)}$.

Corollary 3. For $c \in \mathbb{Z}_{>1}$, then any rational number $\frac{a}{b}$ and $b \neq 0$, has the following representation by a sum of the two cube roots

$$
\begin{aligned}
\frac{a}{b}=\sqrt[3]{-\frac{1}{2}\left[\sqrt{\left.\frac{a^{6}(c+1)^{2}}{b^{6} c^{4}}-\frac{4 a^{6}\left(c^{2}+c+1\right)^{3}}{27 b^{6} c^{6}}+\frac{a^{3}(c+1)}{b^{3} c^{2}}\right]}\right.} \\
+\sqrt[3]{\frac{1}{2}\left[\sqrt{\frac{a^{6}(c+1)^{2}}{b^{6} c^{4}}-\frac{4 a^{6}\left(c^{2}+c+1\right)^{3}}{27 b^{6} c^{6}}}-\frac{a^{3}(c+1)}{b^{3} c^{2}}\right]} .
\end{aligned}
$$

Proof. Let $a \rightarrow \frac{a}{b}$ in Theorem 1, this completes the proof. $\square$

\section{Examples}

For $a=2, b=3$ and $c=2$, then

$\frac{2}{3}=\sqrt[3]{\frac{1}{2}\left(-\frac{2}{9}+\frac{20 i}{81 \sqrt{3}}\right)}+\sqrt[3]{\frac{1}{2}\left(-\frac{2}{9}-\frac{20 i}{81 \sqrt{3}}\right)} ;$

for $a=2, b=3$ and $c=3$, then 
$\frac{2}{3}=\sqrt[3]{\frac{1}{2}\left(-\frac{32}{243}+\frac{560 i}{2187 \sqrt{3}}\right)}+\sqrt[3]{\frac{1}{2}\left(-\frac{32}{243}-\frac{560 i}{2187 \sqrt{3}}\right)}$

for $a=1, b=3$ and $c=2$, then

$\frac{1}{3}=\sqrt[3]{\frac{1}{2}\left(-\frac{1}{36}+\frac{5 i}{162 \sqrt{3}}\right)}+\sqrt[3]{\frac{1}{2}\left(-\frac{1}{36}-\frac{5 i}{162 \sqrt{3}}\right)}$

for $a=1, b=3$ and $c=3$, then

$\frac{1}{3}=\sqrt[3]{\frac{1}{2}\left(-\frac{4}{243}+\frac{70 i}{2187 \sqrt{3}}\right)}+\sqrt[3]{\frac{1}{2}\left(-\frac{4}{243}-\frac{70 i}{2187 \sqrt{3}}\right)}$.

Theorem 2. For $c \in \mathbb{Z}_{\geq 1}$, then any integer a has the following representation by the sum of the two cube roots

$$
a=\sqrt[3]{\frac{1}{2}\left(\frac{a^{3}}{c}+\sqrt{\frac{a^{6}}{c^{2}}-\frac{4 a^{6}(c-1)^{3}}{27 c^{3}}}\right)}+\sqrt[3]{\frac{1}{2}\left(\frac{a^{3}}{c}-\sqrt{\left.\frac{a^{6}}{c^{2}}-\frac{4 a^{6}(c-1)^{3}}{27 c^{3}}\right)}\right.} .
$$

Proof. By Cardano's formula, a root of $x^{3}+p x+q=0$ is given by

$$
x_{1}=\sqrt[3]{\frac{1}{2}\left(-q+\sqrt{q^{2}+\frac{4 p^{3}}{27}}\right)}+\sqrt[3]{\frac{1}{2}\left(-q-\sqrt{q^{2}+\frac{4 p^{3}}{27}}\right)} .
$$

Suppose that $x=\frac{a}{c}$, thus,

$$
x^{3}=-p x-q
$$

By Corollary 2, we obtain

$$
-a c^{2} x^{2}-\left(a^{2}+c^{2} p\right) x+a^{3}-c^{2} q=0,
$$

by Baskara's formula

$$
x=-\frac{a^{2}+c^{2} p \pm \sqrt{\left(a^{2}+c^{2} p\right)^{2}+4 a c^{2}\left(a^{3}-c^{2} q\right)}}{2 a c^{2}} .
$$

By Corollary 3, we encounter

$$
\begin{aligned}
c^{3} x(-p x-q)-a c^{3}(-p x-q)-a^{3} x+a^{4}=0, \\
-c^{3} p x^{2}+\left(a c^{3} p-c^{3} q-a^{3}\right) x+a^{4}+a c^{3} q=0 .
\end{aligned}
$$

Again, by Baskara's formula

$$
x=-\frac{a^{3}+c^{3} q-a c^{3} p \pm \sqrt{\left(a c^{3} p-c^{3} q-a^{3}\right)^{2}+4 c^{3} p\left(a^{4}+a c^{3} q\right)}}{2 c^{3} p}
$$


Compare (7) with (8), and we find

$$
\frac{a^{2}+c^{2} p}{2 a c^{2}}=\frac{a^{3}+c^{3} q-a c^{3} p}{2 c^{3} p}
$$

and

$$
\begin{gathered}
\frac{\sqrt{\left(a^{2}+c^{2} p\right)^{2}+4 a c^{2}\left(a^{3}-c^{2} q\right)}}{2 a c^{2}}=\frac{\sqrt{\left(a c^{3} p-c^{3} q-a^{3}\right)^{2}+4 c^{3} p\left(a^{4}+a c^{3} q\right)}}{2 c^{3} p} \\
\frac{\left(a^{2}+c^{2} p\right)^{2}+4 a c^{2}\left(a^{3}-c^{2} q\right)}{4 a^{2} c^{4}}=\frac{\left(a c^{3} p-c^{3} q-a^{3}\right)^{2}+4 c^{3} p\left(a^{4}+a c^{3} q\right)}{4 c^{6} p^{2}} .
\end{gathered}
$$

Solving the system of equations above, we get

$$
p=-\frac{a^{2}(c-1)}{c}
$$

and

$$
q=-\frac{a^{3}}{c}
$$

or

$$
p=-\frac{a^{2}\left(c^{2}+c+1\right)}{c^{2}}
$$

and

$$
q=\frac{a^{3}(c+1)}{c^{2}}
$$

The solutions (12) and (13) are equals to solutions (5) and (6); thereof, we replace (10) and (11) in (7), and let $x_{1}=a$, this completes the proof.

\section{Examples}

For $a=3$ and $c=2$, then

$3=\sqrt[3]{\frac{1}{2}\left(\frac{27}{2}+\frac{15 \sqrt{3}}{2}\right)}+\sqrt[3]{\frac{1}{2}\left(\frac{27}{2}-\frac{15 \sqrt{3}}{2}\right)} ;$

for $a=3$ and $c=5$, then

$3=\sqrt[3]{\frac{1}{2}\left(\frac{27}{5}+\frac{33}{5} i \sqrt{\frac{3}{5}}\right)}+\sqrt[3]{\frac{1}{2}\left(\frac{27}{5}-\frac{33}{5} i \sqrt{\frac{3}{5}}\right)}$

for $a=4$ and $c=2$, then

$4=\sqrt[3]{\frac{1}{2}\left(32+\frac{160}{3 \sqrt{3}}\right)}+\sqrt[3]{\frac{1}{2}\left(32-\frac{160}{3 \sqrt{3}}\right)} ;$ 
for $a=5$ and $c=2$, then

$5=\sqrt[3]{\frac{1}{2}\left(\frac{125}{2}+\frac{625}{6 \sqrt{3}}\right)}+\sqrt[3]{\frac{1}{2}\left(\frac{125}{2}-\frac{625}{6 \sqrt{3}}\right)}$

for $a=4$ and $c=5$, then

$4=\sqrt[3]{\frac{1}{2}\left(\frac{64}{5}+\frac{704 i}{15 \sqrt{15}}\right)}+\sqrt[3]{\frac{1}{2}\left(\frac{64}{5}-\frac{704 i}{15 \sqrt{15}}\right)} ;$

for $a=4$ and $c=3$, then

$4=\sqrt[3]{\frac{1}{2}\left(-\frac{256}{9}+\frac{4480 i}{81 \sqrt{3}}\right)}+\sqrt[3]{\frac{1}{2}\left(-\frac{256}{9}-\frac{4480 i}{81 \sqrt{3}}\right)}$

for $a=4$ and $c=7$, then

$4=\sqrt[3]{\frac{1}{2}\left(\frac{64}{7}+\frac{320 i}{7 \sqrt{7}}\right)}+\sqrt[3]{\frac{1}{2}\left(\frac{64}{7}-\frac{320 i}{7 \sqrt{7}}\right)} ;$

for $a=5$ and $c=8$, then

$5=\sqrt[3]{\frac{1}{2}\left(\frac{125}{8}+\frac{2125 i}{24 \sqrt{6}}\right)}+\sqrt[3]{\frac{1}{2}\left(\frac{125}{8}-\frac{2125 i}{24 \sqrt{6}}\right)}$.

Corollary 4. For $c \in \mathbb{Z}_{\geq 1}$, then any rational number $\frac{a}{b}$ and $b \neq 0$, has the following representation by the sum of the two cube roots

$\frac{a}{b}=\sqrt[3]{\frac{1}{2}\left[\frac{a^{3}}{b^{3} c}-\sqrt{\frac{a^{6}}{b^{6} c^{2}}-\frac{4 a^{6}(c-1)^{3}}{27 b^{6} c^{3}}}\right]}+\sqrt[3]{\frac{1}{2}\left[\frac{a^{3}}{b^{3} c}+\sqrt{\frac{a^{6}}{b^{6} c^{2}}-\frac{4 a^{6}(c-1)^{3}}{27 b^{6} c^{3}}}\right]}$

Proof. Let $a \rightarrow \frac{a}{b}$ in Theorem 2, this completes the proof. $\square$

\section{Examples}

For $a=1, b=3$ and $c=2$, then

$\frac{1}{3}=\sqrt[3]{\frac{1}{2}\left(\frac{1}{54}+\frac{5}{162 \sqrt{3}}\right)}+\sqrt[3]{\frac{1}{2}\left(\frac{1}{54}-\frac{5}{162 \sqrt{3}}\right)} ;$

for $a=1, b=4$ and $c=2$, then

$\frac{1}{4}=\sqrt[3]{\frac{1}{2}\left(\frac{1}{128}+\frac{5}{384 \sqrt{3}}\right)}+\sqrt[3]{\frac{1}{2}\left(\frac{1}{128}-\frac{5}{384 \sqrt{3}}\right)} ;$

for $a=1, b=5$ and $c=2$, then 
$\frac{1}{5}=\sqrt[3]{\frac{1}{2}\left(\frac{1}{250}+\frac{1}{150 \sqrt{3}}\right)}+\sqrt[3]{\frac{1}{2}\left(\frac{1}{250}-\frac{1}{150 \sqrt{3}}\right)}$

for $a=1, b=6$ and $c=2$, then

$\frac{1}{6}=\sqrt[3]{\frac{1}{2}\left(\frac{1}{432}+\frac{5}{1296 \sqrt{3}}\right)}+\sqrt[3]{\frac{1}{2}\left(\frac{1}{432}-\frac{5}{1296 \sqrt{3}}\right)}$.

Corollary 5. For $c \in \mathbb{Z}_{\geq 1}$, then

$$
1=\sqrt[3]{\frac{1}{2}\left(\frac{1}{c}+\sqrt{\frac{1}{c^{2}}-\frac{4(c-1)^{3}}{27 c^{3}}}\right)}+\sqrt[3]{\frac{1}{2}\left(\frac{1}{c}-\sqrt{\frac{1}{c^{2}}-\frac{4(c-1)^{3}}{27 c^{3}}}\right)} .
$$

Proof. Simplifying the right-hand side of Theorem 2 and dividing both members for $a$. $\square$

\section{Examples}

For $c=2$,

$$
1=\sqrt[3]{\frac{1}{2}\left(\frac{1}{2}+\frac{5}{6 \sqrt{3}}\right)}+\sqrt[3]{\frac{1}{2}\left(\frac{1}{2}-\frac{5}{6 \sqrt{3}}\right)}
$$

for $c=5$,

$$
1=\sqrt[3]{\frac{1}{2}\left(\frac{1}{5}+\frac{11 i}{15 \sqrt{15}}\right)}+\sqrt[3]{\frac{1}{2}\left(\frac{1}{5}-\frac{11 i}{15 \sqrt{15}}\right)}
$$

for $c=7$,

$$
1=\sqrt[3]{\frac{1}{2}\left(\frac{1}{7}+\frac{5 i}{7 \sqrt{7}}\right)}+\sqrt[3]{\frac{1}{2}\left(\frac{1}{7}-\frac{5 i}{7 \sqrt{7}}\right)} .
$$

Lemma 3. For $c \in \mathbb{Z}_{>1}$, then

$$
1=\sqrt[3]{\frac{1}{2}\left(-\frac{c+1}{c^{2}}+\sqrt{\frac{(c+1)^{2}}{c^{4}}-\frac{4\left(c^{2}+c+1\right)^{3}}{27 c^{6}}}\right)}+\sqrt[3]{\frac{1}{2}\left(-\frac{c+1}{c^{2}}-\sqrt{\frac{(c+1)^{2}}{c^{4}}-\frac{4\left(c^{2}+c+1\right)^{3}}{27 c^{6}}}\right)} .
$$

Proof. Simplifying the right-hand side of Theorem 1 and dividing both members for $a$.

Theorem 4. For $n \in \mathbb{Z}_{>1}$, then $n$ has the following representation by the sum of cube roots

$$
\begin{gathered}
n=\sum_{k=2}^{n+1}\left[\sqrt[3]{\frac{1}{2}\left(-\frac{k+1}{k^{2}}+\sqrt{\left.\frac{(k+1)^{2}}{k^{4}}-\frac{4\left(k^{2}+k+1\right)^{3}}{27 k^{6}}\right)}\right.}\right. \\
+\sqrt[3]{\frac{1}{2}\left(-\frac{k+1}{k^{2}}-\sqrt{\left.\frac{(k+1)^{2}}{k^{4}}-\frac{4\left(k^{2}+k+1\right)^{3}}{27 k^{6}}\right)}\right.} .
\end{gathered}
$$

Proof. Using the Lemma 3 and finite induction, this completes the proof. $\square$ 


\section{Examples}

$$
\begin{aligned}
& 1=\sqrt[3]{\frac{1}{2}\left(-\frac{3}{4}+\frac{5 i}{6 \sqrt{3}}\right)}+\sqrt[3]{\frac{1}{2}\left(-\frac{3}{4}-\frac{5 i}{6 \sqrt{3}}\right)} ; \\
& 2=\sqrt[3]{\frac{1}{2}\left(-\frac{3}{4}+\frac{5 i}{6 \sqrt{3}}\right)}+\sqrt[3]{\frac{1}{2}\left(-\frac{3}{4}-\frac{5 i}{6 \sqrt{3}}\right)}+\sqrt[3]{\frac{1}{2}\left(-\frac{4}{9}+\frac{70 i}{81 \sqrt{3}}\right)}+\sqrt[3]{\frac{1}{2}\left(-\frac{4}{9}-\frac{70 i}{81 \sqrt{3}}\right)} ; \\
& 3=\sqrt[3]{\frac{1}{2}\left(-\frac{3}{4}+\frac{5 i}{6 \sqrt{3}}\right)}+\sqrt[3]{\frac{1}{2}\left(-\frac{3}{4}-\frac{5 i}{6 \sqrt{3}}\right)}+\sqrt[3]{\frac{1}{2}\left(-\frac{4}{9}+\frac{70 i}{81 \sqrt{3}}\right)}+\sqrt[3]{\frac{1}{2}\left(-\frac{4}{9}-\frac{70 i}{81 \sqrt{3}}\right)} \\
& +\sqrt[3]{\frac{1}{2}\left(-\frac{5}{16}+\frac{9 i \sqrt{3}}{32}\right)}+\sqrt[3]{\frac{1}{2}\left(-\frac{5}{16}-\frac{9 i \sqrt{3}}{32}\right)} ; \\
& 4=\sqrt[3]{\frac{1}{2}\left(-\frac{3}{4}+\frac{5 i}{6 \sqrt{3}}\right)}+\sqrt[3]{\frac{1}{2}\left(-\frac{3}{4}-\frac{5 i}{6 \sqrt{3}}\right)}+\sqrt[3]{\frac{1}{2}\left(-\frac{4}{9}+\frac{70 i}{81 \sqrt{3}}\right)}+\sqrt[3]{\frac{1}{2}\left(-\frac{4}{9}-\frac{70 i}{81 \sqrt{3}}\right)} \\
& +\sqrt[3]{\frac{1}{2}\left(-\frac{5}{16}+\frac{9 i \sqrt{3}}{32}\right)}+\sqrt[3]{\frac{1}{2}\left(-\frac{5}{16}-\frac{9 i \sqrt{3}}{32}\right)}+\sqrt[3]{\frac{1}{2}\left(-\frac{6}{25}+\frac{308 i}{375 \sqrt{3}}\right)}+\sqrt[3]{\frac{1}{2}\left(-\frac{6}{25}-\frac{308 i}{375 \sqrt{3}}\right)} .
\end{aligned}
$$

